\title{
Fingering Instabilities in Dewetting Nanofluids
}

\author{
E. Pauliac-Vaujour, A. Stannard, C. P. Martin, M. O. Blunt, I. Notingher, and P. J. Moriarty \\ The School of Physics and Astronomy, The University of Nottingham, Nottingham NG7 2RD, United Kingdom \\ I. Vancea and U. Thiele \\ School of Mathematics, Loughborough University, Leicestershire LE11 3TU, United Kingdom \\ Max-Planck-Institut für Physik komplexer Systeme, Nöthnitzer Strasse 38, 01187 Dresden, Germany
}

(Received 18 January 2008; published 30 April 2008)

\begin{abstract}
The growth of fingering patterns in dewetting nanofluids (colloidal solutions of thiol-passivated gold nanoparticles) has been followed in real time using contrast-enhanced video microscopy. The fingering instability on which we focus here arises from evaporatively driven nucleation and growth in a nanoscopically thin precursor solvent film behind the macroscopic contact line. We find that well-developed isotropic fingering structures only form for a narrow range of experimental parameters. Numerical simulations, based on a modification of the Monte Carlo approach introduced by Rabani et al. [Nature (London) 426, 271 (2003)], reproduce the patterns we observe experimentally.
\end{abstract}

A remarkable variety of systems in nature form striking branched structures which exhibit fractal properties [1,2]. Key examples include river beds [3], snowflakes [4], bacterial populations [2], and the "viscous fingering" patterns which form at the interface of fluids of different viscosity [5]. The development of fingering instabilities in dewetting thin films [6-11], an area of significant topical interest, has particular potential in the context of self-organized nanostructures. A number of groups have recently exploited fingering instabilities in drying nanofluids to form linear assemblies of particles [10,11] exhibiting an impressive degree of pseudo-one-dimensional order. In each case, the instability occurs at the three phase contact line of the dewetting colloidal solution. Monte Carlo simulations [12], however, predict the formation of a different class of fingering structure in dewetting nanofluids which forms not at the macroscopic contact line but at the edges of thermally nucleated holes in a nanoscopically thin solvent film.

Here we describe the results of a systematic study of fingering instabilities in dewetting films of the "archetypal" nanofluid: a solution of thiol-passivated Au nanoparticles in an organic solvent. We show that fingering patterns can arise both from transverse instabilities at the macroscopic solvent-air-substrate contact line and from the interplay of solvent and nanoparticle dynamics in a microscopic ("precursor") solvent film behind the macroscopic dewetting front. Experimental realization of the latter, however, necessitates careful tuning of nanoparticle mobility (via the thiol chain length) and the dynamics of solvent dewetting. A simple modification of the Hamiltonian introduced by Rabani et al. [13] reproduces the experimentally observed patterns numerically.

In our experiments, solutions of $2 \mathrm{~nm}$ thiol-passivated gold nanoparticles in either toluene or dichloromethane [14] are deposited onto native oxide-terminated $\mathrm{Si}(111)$ substrates. Following solvent evaporation, the nanoparticles form a broad variety of self-organized patterns on the surface [15-18]. We carried out the deposition using a simple strategy which exploits the affinity of the solvent with a Teflon ring to form a meniscus of solution [19]. Evaporation times within the ring vary from a few tens of seconds (at the center) up to a few hours (at the edge) [19] and thus, on a single sample, the system can be controllably varied between the "homogeneous" and "heterogeneous" limits of solvent evaporation discussed by Rabani et al. [13]. Real-time observations of nanoparticle selforganization and the motion of the dewetting front were achieved using the Sarfus contrast-enhanced microscopy technique commercially available from Nanolane [20]. Tapping mode atomic force microscopy (AFM) (Asylum Research MFP-3D) was used to image nanoparticle distributions following evaporation of the solvent.

Figure 1(a) is taken from video 1 of Ref. [21] and shows the formation of two types of fingering pattern: large-scale anisotropic (elongated) fingers surrounded by smaller more isotropic branched structures. These are both seen somewhat more clearly in the 3-dimensional representation in Fig. 1(b). Video 1 provides key insights into the finger formation mechanisms. The large-scale fingers result from a transverse instability at the contact line which is on first consideration apparently similar to that which underlies the formation of fingers in the experiments of $\mathrm{Xu}$ et al. [10] and Huang et al. [11]. Note, however, that at a slower front velocity (in the latter half of the video), there are no fingering instabilities at the macroscopic dewetting front. We return to this point below.

Accompanying the instability at the macroscopic front is the formation of isotropic fingered structures a significant distance behind the retracting contact line. It has previously been postulated that the essential dynamics of pattern formation in many nanofluid systems occurs in an ultrathin 

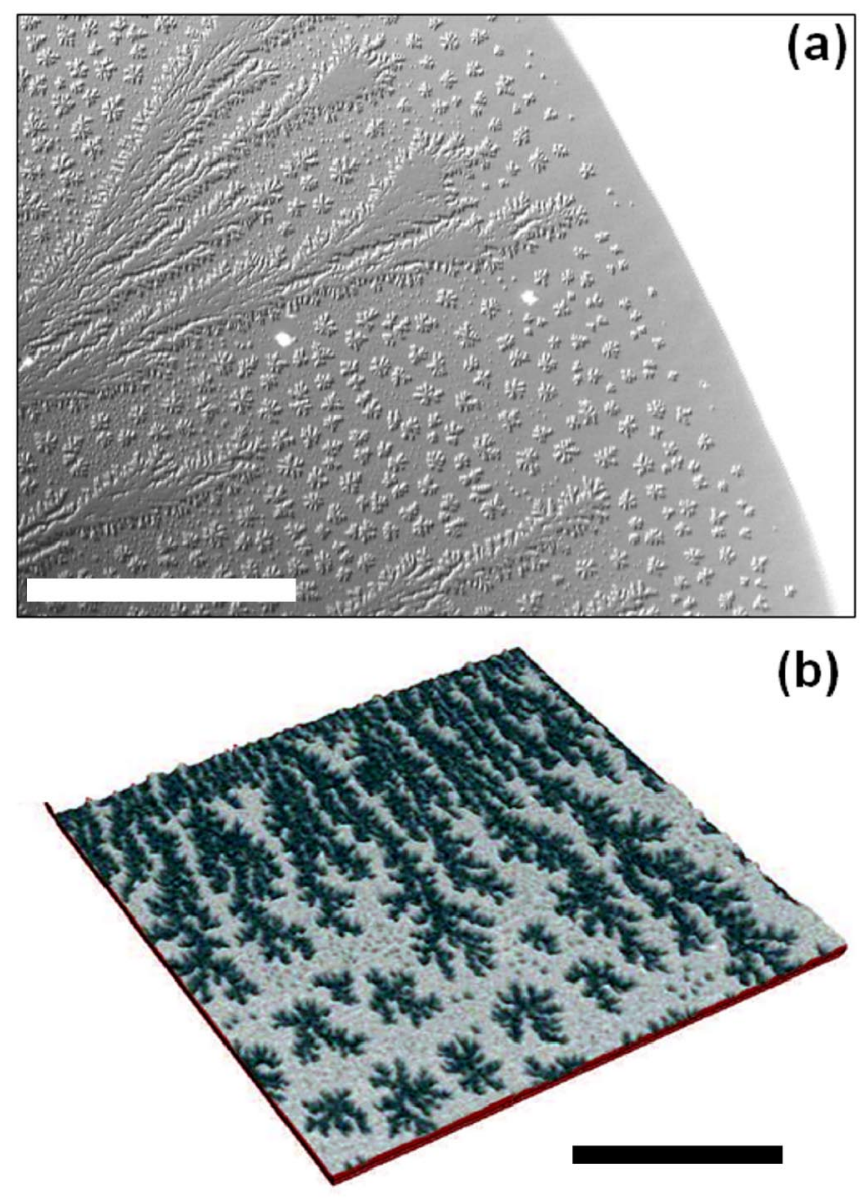

FIG. 1 (color online). (a) Capture from video 1 [21] showing the formation of fingered structures at the substrate-solvent interface as the dewetting front retreats. The white region in the top right-hand corner is the retracting macroscopic solvent film. Note the formation of isotropic branched structures behind the macroscopic contact line. Scale bar: $200 \mu \mathrm{m}$. (b) 3D view of the resulting fingering patterns. The layer is one nanoparticle thick in each case. Scale bar: $50 \mu \mathrm{m}$.

solvent film (and can therefore be modeled effectively by 2D [13] or pseudo-3D [18] simulations). Video 1 represents the first direct experimental verification that this is indeed the case.

The formation of well-developed isotropic fingering patterns of the type observed in Fig. 1 is possible only within a relatively narrow range of experimental parameters. Specifically, for nanoparticle solutions in toluene, relatively low mobility nanoparticles (see discussion below), a particle concentration of $0.1-1.0 \mathrm{mg} / \mathrm{mL}$, and a solvent evaporation time between 1 and $20 \mathrm{~min}$ are required. Moreover, modifying the thiol chain length will change interparticle and particle-substrate interactions which both contribute to the effective diffusion barrier for the particle in solution. A relatively straightforward method of modifying nanoparticle mobility therefore is to change the length of the thiol group coating the particle (at fixed core diameter $\sim 2 \mathrm{~nm}$ ). We show in Figs. 2(a)2(e) AFM images of submonolayer coverages of thiol- passivated $\mathrm{Au}$ nanoparticles on native oxide-terminated $\mathrm{Si}(111)$ where the chain length has been varied systematically from pentane $\left(C_{5}\right)$ to tetradecane $\left(C_{14}\right)$. The nanoparticle concentration (in toluene) and deposited volume were kept fixed. For $C_{5}$ and $C_{8}$ ligands we have not observed the formation of fingered structures at any position within the ring geometry [19]; i.e., irrespective of solvent evaporation time, fingered structures do not form for short chain lengths.

With $C_{10}$ and $C_{12}$ [Figs. 2(c) and 2(d), respectively], well-developed fingered structures are formed. Increasing the chain length to $C_{14}$ [Fig. 2(e)], however, tends not to improve the development of the fingered structures further and, indeed, can lead to more poorly defined branches. (This is more clearly observed for the nanoparticle solutions containing an excess of thiol discussed below.) The results of Figs. 2(a)-2(e) may be understood in terms of the changes in nanoparticle mobility which arise from differences in chain length and the interpenetration of the thiol ligands. That we observe branched structures only for $C_{10^{-}}, C_{12^{-}}$, and $C_{14^{-}}$passivated nanoparticles is most likely due to a lack of interdigitation-i.e., the interlocking of bundles of the passivating molecules [22] - for shorter chains [23,24]. In addition, and as highlighted by Motte and Pileni [23], end gauche defects in $C_{14}$ chains tend to produce less pronounced interdigitation than for $C_{12}$ thiols - a plausible origin of the less-developed fingering for $C_{14}$ [Figs. 2(e) and 2(j)]. A sufficiently low mobility, coupled with appropriate evaporation dynamics [12], leads to the formation of well-defined fingers. Moreover, and of particular significance with regard to the Monte Carlo simulations discussed below, the isotropic fingered structures are invariably surrounded by a high number density of very small holes [see Figs. 2(c) and 2(d)].

Addition of an excess of $0.1 \%$ of thiol by volume [25] to each of the solutions used to prepare the samples shown in Figs. 2(a)-2(e) dramatically enhances the development of fingered structures [see Figs. 2(f) $-2(\mathrm{j})$ ]. In the case of $C_{5^{-}}$ and $C_{8}$-passivated particles [Figs. 2(f) and 2(g)], fingered features, albeit sometimes poorly defined, are now observed, in contrast to the case without excess thiol [Figs. 2(a) and 2(b)]. For $C_{10}, C_{12}$, and $C_{14}$ [Figs. 2(h)2(j)], the fingering is considerably enhanced and patterns spreading over tens of microns are observed. Excess thiol increases the viscosity of the colloidal solution, reducing the nanoparticle mobility and enhancing the development of fingered structures [21]. The addition of excess thiol also increases the time the solvent requires to start to dewet. It does not, however, strongly affect the solvent evaporation rate following the initiation of dewetting [19].

With appropriate parameters, fingered structures appear in Monte Carlo simulations using the 2D model of Rabani et al. [13] or its 3D counterpart [12]. The original 2D code [13] does not, however, reproduce the coexisting large isotropic well-separated fingered structures and surrounding high-density distribution of smaller holes which are 

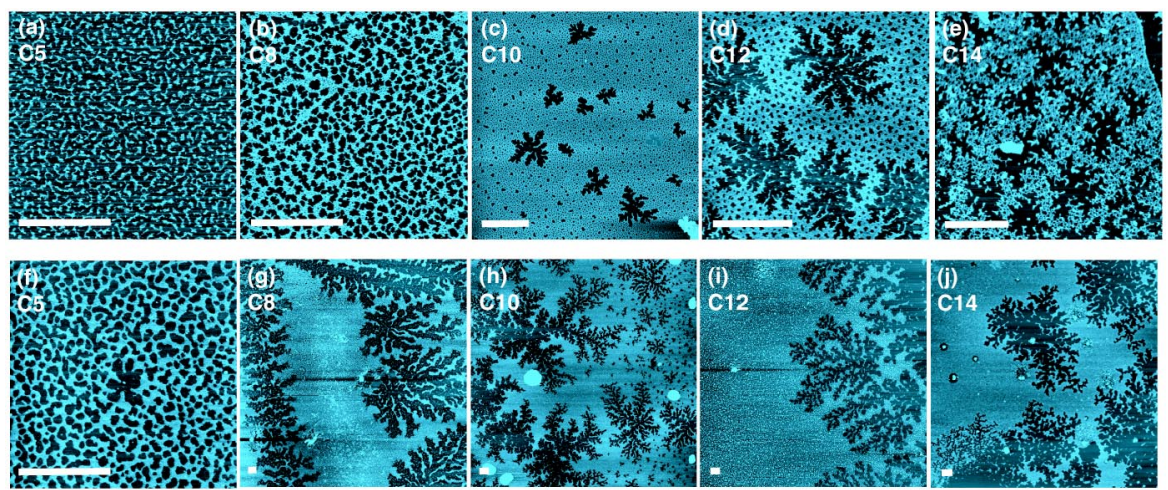

FIG. 2 (color online). AFM scans of (a),(f) $C_{5^{-}},(\mathrm{b}),(\mathrm{g}) C_{8^{-}},(\mathrm{c}),(\mathrm{h}) C_{10^{-}}$, (d),(i) $C_{12^{-}}$, and (e),(j) $C_{14^{-}}$thiol-passivated gold nanoparticles deposited from toluene onto native oxide-terminated $\mathrm{Si}(111)$. Samples (a)-(e) were prepared without excess thiol, samples (f)-(j) with solutions containing $0.1 \%$ excess thiol by volume. All scale bars represent $2 \mu \mathrm{m}$.

always observed in our experiments. This significant experimental observation strongly suggests that two separate regimes of dewetting occur. Furthermore, given that video 1 clearly illustrates that fingering instabilities of the type shown in Fig. 2 develop in a thin solvent film, an important question is whether a pseudo-2D model [18] could reproduce the observed bimodal patterns. We have therefore taken the original Rabani et al. Hamiltonian [13]

$$
E=-\epsilon_{l} \sum_{\langle i j\rangle} l_{i} l_{j}-\epsilon_{n} \sum_{\langle i j\rangle} n_{i} n_{j}-\epsilon_{n l} \sum_{\langle i j\rangle} n_{i} l_{j}-\mu(\nu) \sum_{i} l_{i},
$$

where $\epsilon_{l}, \epsilon_{n}$, and $\epsilon_{n l}$ are the liquid-liquid, nanoparticlenanoparticle, and nanoparticle-liquid interaction energies, respectively, and made the chemical potential $\mu$ a function of the global solvent coverage $\nu$ as in Martin et al. [18]. This enables 3D solvent behavior to be incorporated within the Hamiltonian but, importantly, maintains the 2D character of the nanoparticle "subsystem." Although in Ref. [18] we used a power law dependence, the dewetting dynamics observed in video 1 strongly suggests that to reproduce the experimental patterns a step-function-like change is more appropriate. We have, therefore, taken the chemical potential function $\mu(\nu)$ to be sigmoidal in form [see inset of Fig. 3(c)], i.e.,

$$
\mu(\nu)=\mu_{0}\left\{1+\frac{\Delta \mu_{f}}{1+\exp \left[\left(\nu-\nu_{s}\right) / \sigma\right]}\right\},
$$

where $\mu_{0}$ is the initial chemical potential and $\Delta \mu_{f}$ is its fractional change. $\nu_{s}$ and $\sigma$ define the position and the width of the step, respectively.

In Fig. 3 we show an experimental image alongside a simulated fingered structure. Qualitatively, the level of agreement is very good. Importantly, in addition to accurately reproducing the morphology of the isolated fingered structure, the simulation also yields the surrounding smaller holes. In the simulation (see video 2 [21]), first a fingering structure forms via nucleation and grows at a given chemical potential. The switch in chemical potential then triggers a spinodal dewetting process in the ultrathin solvent film surrounding the fingered structure which in turn leads to the appearance of many small holes in the solvent film within a narrow time window. As is clear from video 2, the holes in Fig. 3(b) appear in what Rabani et al. [13] term the "homogeneous evaporation" regime and, as in the experimental image, thus have a rather narrow distribution of diameters. A similar competition between

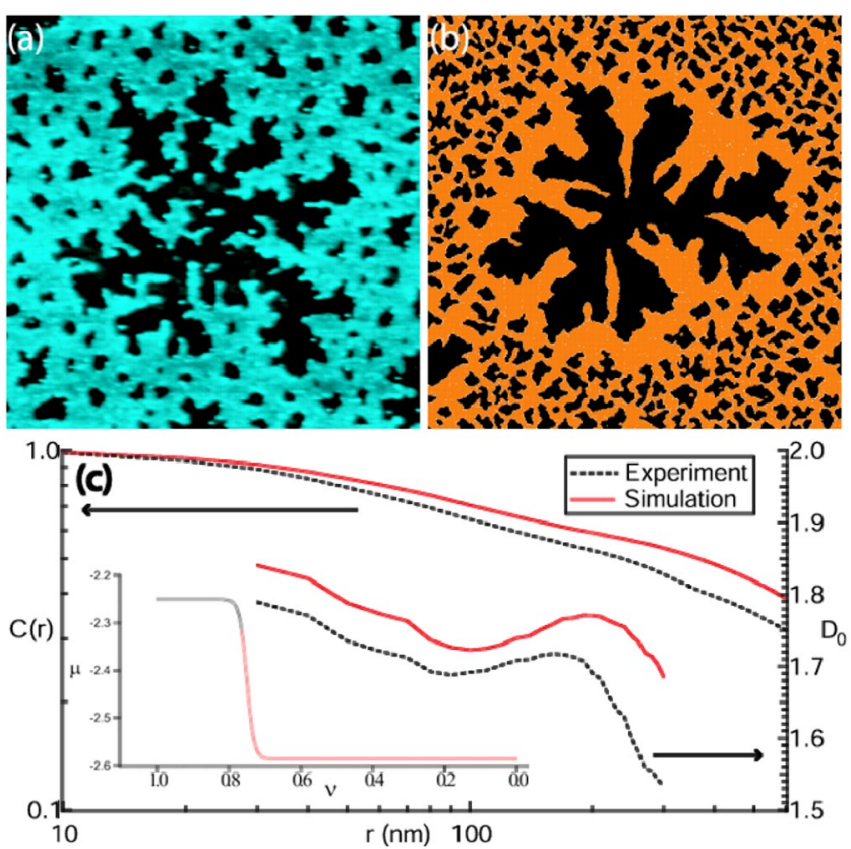

FIG. 3 (color online). (a) AFM image of a fingering instability formed in a nanofluid comprising dodecanethiol-passivated $\mathrm{Au}$ nanoparticle. (b) A simulated image using $\mu_{0}=-2.25 \epsilon_{l}$, $\Delta \mu_{f}=0.15, \nu_{s}=0.75$, and $\sigma=0.01$ [see Eq. (2)]. (c) The plots in the upper half of the graph are the averaged correlation function for four different fingering structures (experiment) and eight fingering structures (simulation). In both cases there is power law behavior at intermediate length scales $(\sim 30$ $200 \mathrm{~nm}$ ). The lower half of the graph shows the variation of fractal dimension with length. Inset: Plot of the sigmoidal dependence of chemical potential (in units of $\epsilon_{l}$ ) on solvent coverage. 
dewetting by nucleation and via a spinodal process was previously discussed for aqueous solutions of collagen [26].

The physical picture which emerges from a comparison of the experimental and simulation data is that the isotropic fingering patterns arise from a transverse instability of the evaporative dewetting front around growing thermally nucleated holes. The instability develops when the nanoparticle mobility is sufficiently low such that the particles are "collected" by the solvent front. When too many are collected they are "expelled" through the instability mechanism and deposited in fingers, a process reminiscent of the auto-optimization of dewetting rates previously described for polymer films [27]. At a later stage, i.e., when the remaining precursor film is even thinner, a spinodal instability triggers the appearance of the high density of smaller holes which surround the fingered structures. The process is caused by additional short-range interactions that become effective at very small film thicknesses [18].

To move beyond a qualitative comparison, it is important to find a robust morphological metric which can be used to analyze the fingering structures. We chose to use the correlation function algorithm for the calculation of fractal dimension [28], as described by Praud and Swinney [29]. (See [21] for a full description). Density-density correlation functions for the experimental and simulated images are given in Fig. 3(c). The average fractal dimension for dodecanethiol-passivated nanoparticle fingered structures $\left\langle D_{0}\right\rangle$ is $1.70 \pm 0.05$ and that for the simulated structures is $1.75 \pm 0.05$. Moreover, there is a close similarity between the behavior of the local slopes [lower half of Fig. 3(c)]. That we obtain good quantitative agreement between the morphological characteristics of the experimental fingered structures and their simulated counterparts, as shown by the similarity of the density-density correlation plots in Fig. 3, suggests that a step change of chemical potential with solvent coverage captures the essential dynamics of the drying solvent and its coupling to the nanoparticle motion.

We have focused here on the fingering patterns which appear some distance behind the macroscopic dewetting front largely because these are amenable to simulation via the Monte Carlo method described above and elsewhere $[13,18]$. Returning to video 1 [21], however, there remain details regarding fingering instabilities at the macroscopic contact line which need to be understood. Given that we observe a contact line instability only for relatively high evaporation rates, the Marangoni effect, as described by Maillard et al. [30], is the most likely origin of the fingering structures which appear at the dewetting front in video 1. Incorporation of the Marangoni effect within the Monte Carlo model described above is challenging and will require a hybrid continuum-microscopic model which we are in the process of developing.

We acknowledge funding by the EU (MRTN-CT-2004005728, PATTERNS). We thank J. Sharp for valuable discussions. E. P. V. thanks P. Croguennoc, N. Médard, and B. Landemaine (Nanolane, Montfort-le-Gesnois) for supporting the real-time imaging.

[1] B. B. Mandelbrot, The Fractal Geometry of Nature (W. H. Freeman, New York, 1983).

[2] P. Ball, The Self-Made Tapestry: Pattern Formation in Nature (Oxford University, New York, 1999).

[3] S. S. Manna and B. Subramanian, Phys. Rev. Lett. 76, 3460 (1996).

[4] H. M. Singer, Phys. Rev. E 73, 051606 (2006), and references therein.

[5] H. J. S. Hele Shaw, Nature (London) 58, 34 (1898).

[6] A. V. Lyushnin, A. A. Golovin, and L. M. Pismen, Phys. Rev. E 65, 021602 (2002).

[7] U. Thiele, Eur. Phys. J. E 12, 409 (2003).

[8] L. V. Govor, G. Reiter, and G. H. Bauer, Phys. Rev. E 74, 061603 (2006).

[9] N. Samid-Merzel, S. G. Lipson, and D. S. Tannhauser, Phys. Rev. E 57, 2906 (1998).

[10] Jun Xu, Jianfeng Xia, and Zhiqun Lin, Angew. Chem., Int. Ed. 46, 1860 (2007).

[11] J. Huang, F. Kim, A. R. Tao, S. Connor, and P. Yang, Nat. Mater. 4, 896 (2005).

[12] G. Yosef and E. Rabani, J. Phys. Chem. B 110, 20965 (2006).

[13] E. Rabani, D. R. Reichman, P. L. Geissier, and L. E. Brus, Nature (London) 426, 271 (2003).

[14] M. Brust et al., J. Chem. Soc. Chem. Commun. 7, 801 (1994).

[15] G. Ge and L. Brus, J. Phys. Chem. B 104, 9573 (2000).

[16] P. Moriarty, M.D. R. Taylor, and M. Brust, Phys. Rev. Lett. 89, 248303 (2002).

[17] M.O. Blunt, et al., Nature Nanotechnology 2, 167 (2007).

[18] C. P. Martin et al., Phys. Rev. Lett. 99, 116103 (2007).

[19] E. Pauliac-Vaujour and P. Moriarty, J. Phys. Chem. C 111, 16255 (2007).

[20] http://www.nano-lane.com.

[21] See EPAPS Document No. E-PRLTAO-100-050818 for supplementary videos. For more information on EPAPS, see http://www.aip.org/pubservs/epaps.html.

[22] W. D. Luedtke, and U. Landman, J. Phys. Chem. 100, 13323 (1996).

[23] L. Motte and M. P. Pileni, Appl. Surf. Sci. 164, 60 (2000).

[24] M. J. Hostetler, J. J. Stokes, and R. W. Murrary, Langmuir 12, 3604 (1996).

[25] X.M. Lin, H.M. Jaeger, C.M. Sorensen, and K.J. Klabunde, J. Phys. Chem. B 105, 3353 (2001).

[26] U. Thiele, M. Mertig, and W. Pompe, Phys. Rev. Lett. 80, 2869 (1998).

[27] G. Reiter and A. Sharma, Phys. Rev. Lett. 87, 166103 (2001).

[28] P. Grassberger and I. Procaccia, Phys. Rev. Lett. 50, 346 (1983).

[29] O. Praud and H. L. Swinney, Phys. Rev. E 72, 011406 (2005).

[30] M. Maillard, L. Motte, and M.-P. Pileni, Adv. Mater. 13, 200 (2001). 